Recibido: 28/08/2020 --- Aceptado: 01/10/2020 --- Publicado: 12/04/2021

\title{
LA ESTRATEGIA DE COMUNICACIÓN DE RSC DE IBERDROLA EN FACEBOOK Y TWITTER: UN ANÁLISIS LINGÜÍSTICO Y DE CONTENIDO BASADO EN CORPUS
}

\section{THE CSR COMMUNICATION STRATEGY OF IBERDROLA ON FACEBOOK AND TWITTER: A CORPUS-BASED LINGUISTIC AND CONTENT ANALYSIS}

Helena Moyaert ${ }^{1}$ : Universiteit Antwerpen. Bélgica.

helena.moyaert@gmail.com

Lieve Vangehuchten: Universiteit Antwerpen. Bélgica. lieve.vangehuchten@uantwerpen.be

Ana M. Fernández Vallejo: Universidad de Navarra. España. amf@unav.es

\section{Cómo citar el artículo:}

Moyaert, H., Vangehuchten, L. y Fernández-Vallejo, A. M. (2021). La estrategia de comunicación de RSC de Iberdrola en Facebook y Twitter: Un análisis lingüístico y de contenido basado en corpus. Revista de Comunicación de la SEECI, 54, 119-143. http://doi.org/10.15198/seeci.2021.54.e664

\section{RESUMEN}

En nuestra civilización digitalizada, en la que el compartir información e interactuar sobre ella está cada vez más a disposición de todos, se necesitan estudios sobre las prácticas de comunicación empresarial de la Responsabilidad Social Corporativa (RSC) en los medios sociales. El presente estudio de corpus contribuye a llenar este vacío mediante un análisis de contenido y lingüístico multimodal de la forma en que una empresa energética del IBEX 35, Iberdrola, comunica sobre su política de RSC en los medios sociales. El corpus consta de 438 posts en Twitter y 126 posts en Facebook. Los resultados permiten sacar las siguientes conclusiones: 1) la estrategia informativa de Iberdrola varía según el canal: en Twitter el enfoque está en medio ambiente, sostenibilidad, inversión social, grupos de interés y artes, mientras que en Facebook se dirige principalmente a los grupos de interés, motivándolos a

1 Helena Moyaert: Máster en Comunicación Profesional Multilingüe (junio 2020) y graduada en Lingüística Aplicada Español e Inglés (junio 2018) por la Universidad de Amberes. helena.moyaert@gmail.com 
Moyaert, H.; Vangehuchten, L y Fernández-Vallejo, A.M. La estrategia de comunicación de RSC de Iberdrola en Facebook y Twitter: Un análisis lingüístico y de contenido basado en

comprometerse con la RSC; 2) existe indudablemente una estrategia interactiva que caracteriza el discurso digital, como las estructuras que suscitan emociones (positivas), el uso de lenguaje informal (los emoticones y el uso de la primera y segunda persona) y otros elementos multimodales como los hashtags y tags, que todos sirven para aumentar la confianza y el compromiso por la marca; 3) hay patrones subyacentes en la estrategia informativa e interactiva, en el sentido de que Iberdrola quiere comunicar su política de RSC a través de un lenguaje informal y emocional (positivo), con la excepción del lenguaje emocional negativo cuando se discuten temas ambientales, muy probablemente para evitar acusaciones de ecoblanqueo.

PALABRAS CLAVE: Responsabilidad Social Corporativa (RSC) - Comunicación empresarial digital - Medios sociales - Sector energético - España - Iberdrola Análisis de contenido - Análisis lingüístico multimodal - Análisis de corpus.

\section{ABSTRACT}

In our increasingly digitalized civilization where sharing information and interacting about it is ever more made available to the general public, research into corporate communication practices of Corporate Social Responsibility (CSR) content on social media is desperately needed. This study makes a contribution to fill this research gap by performing a corpus-based content and multimodal linguistic analysis of the way in which an IBEX 35 energy company, Iberdrola, communicates about its CSR policy on social media. The corpus consists of 438 posts on Twitter and 126 posts on Facebook. The results allow to draw the following conclusions: 1) Iberdrola's information strategy varies from social media channel, where on Twitter the focus is through the environment, sustainability, social investment, stakeholders and arts, and on Facebook the focus is directed at stakeholders, thus motivating them to engage with CSR-related information; 2) there is certainly an interactive strategy, with many elements that characterize digital discourse, such as structures that elicit (positive) emotions, informal language use (like emojis and use of 1st and 2nd person) and other multimodal elements like hashtags and tags, which are all efforts at increasing brand trust, engagement, and admiration; 3) there are some underlying patterns within the informative and the interactive strategy, being that the overall way in which Iberdrola wants to communicate its CSR information is through informal and (positive) emotional language, with an exception of negative emotional language when discussing environmental themes, most probably to avoid accusations of greenwashing.

KEYWORDS: Corporate Social Responsibility (CSR) - Digital corporate communication - Social media - Energy sector - Spain - Iberdrola - Content-based analysis - Multimodal linguistic analysis - Corpus analysis.

\section{A ESTRATÉGIA DE COMUNICAÇÃO DA RSC DE IBERDROLA NO FACEBOOK E TWITTER: UMA ANALISE LINGUÍSTICA E DE}




\section{CONTEÚDO BASEADO EM CORPUS}

\section{RESUMO:}

Na nossa civilização digital, na que compartilhar informação e interagir sobre ela está cada vez mais à disposição de todos, é preciso estudar sobre as práticas de comunicação empresarial da Responsabilidade Social Corporativa (RSC) nos meios sociais. O presente estudo de corpus contribui a preencher este vazio através de uma análise de conteúdo linguístico multimodal da forma na que uma empresa de energia do IBEX 35, Iberdrola, comunica sobre sua política de RSC nos meios sociais. O corpus contém 438 posts no Twitter e 126 posts no Facebook.

Os resultados permitem tirar as seguintes conclusões: 1) a estratégia informativa de Iberdrola varia de acordo com o canal: no Twitter o foco está no meio ambiente, sustentabilidade, investimento social, grupos de interesse e artes, enquanto que no Facebook aborda principalmente os grupos de interesse, motivando-os a se comprometer com a RSC; 2) sem dúvida existe uma estratégia interativa que caracteriza o discurso digital, tais como as estruturas que despertam emoções (positivas), o uso de linguagem informal (os emoticons e o uso da primeira e segunda pessoa) e outros elementos multimodais como os hashtags e tags, que servem para aumentar a confiança e o comprometimento pela marca; 3) existem padrões subjacentes na estratégia informativa e interativa, no sentido de que Iberdrola quer comunicar sua política de RSC a través de uma linguagem informal e emocional (positiva), com a exceção de linguagem emocional negativa quando se discutem temas ambientais, muito provavelmente para evitar acusações de eco branqueamento.

Palavras chave: Responsabilidade Social Corporativa (RSC) - Comunicação empresarial digital - Meios sociais - Setor energético - Espanha - Iberdrola - Análise de conteúdo - Análise lingüístico multimodal - Análise de corpus.

Traducido por Paula González (Universidad Católica Andrés Bello, Venezuela)

\section{INTRODUCCIÓN}

Tanto la comunicación de RSC y la comunicación en redes sociales se han convertido en pilares importantes en una sociedad cada vez más compleja y consciente. La demanda por parte de la sociedad y los accionistas por una buena comunicación de RSC está creciendo día a día, y la comprensión de que tenemos un compromiso con nuestro planeta es simplemente innegable (Bonsón y Ratkai, 2013). Sin embargo, una investigación reciente de Postma (2015), muestra que a menudo hay desacuerdo e incertidumbre sobre la idoneidad de las redes sociales para comunicar la RSC y que hay necesidad de consejos concretos y un marco de referencia claro. 
Moyaert, H.; Vangehuchten, L y Fernández-Vallejo, A.M. La estrategia de comunicación de RSC de Iberdrola en Facebook y Twitter: Un análisis lingüístico y de contenido basado en corpus

En el caso de la RSC en España, hay ciertas pautas que establecen una estrategia de RSC, como la Ley $11 / 2018^{2}$, pero no especifican nada en el área de las redes sociales. Para ello, este artículo examina las publicaciones de Facebook y Twitter de Iberdrola, una multinacional española que opera en el sector eléctrico. Esta multinacional fue galardonada con el Premio a la Mejor Gobernación Corporativa en España 2019, y analizar las estrategias de comunicación de la firma puede llevar a conocimientos relevantes que permitirán a empresas construir un mejor marco para la RSC en las redes sociales en el futuro. Además, según un estudio llevado a cabo por Epsilon, Iberdrola ocupa un lugar destacado en el manejo de sus redes sociales. Tanto las publicaciones de Facebook y Twitter se analizan porque estos canales tienen un número elevado y similar de seguidores y el contenido de RSC se publica en ambos canales.

En resumen, la RSC en la actualidad es un tema de alto perfil para las empresas y desde esa perspectiva, este artículo quiere dar respuesta a la pregunta de cómo una empresa de habla hispana cumple con su política de RSC a través de redes sociales, principalmente en términos de contenido y potencial interactivo. Más precisamente, la pregunta principal de investigación (PI) que se abordará es:

PI "¿Cómo cumple una empresa española su estrategia comunicativa en torno a la RSC en las redes sociales?"

Las sub- preguntas que se abordarán son:

PI1 "¿Qué temas relacionados con la RSC se utilizan (con mayor frecuencia)?"; PI2 "¿Existe un posible patrón discursivo en la forma en que se formulan los mensajes, más precisamente en cuanto al uso del lenguaje emocional, lenguaje informal y elementos multimodales?"

PI3 "¿Se combinan ciertos temas con ciertos patrones textuales?"

A continuación, primero comentamos con más profundidad la situación actual de la comunicación de RSC en España. Luego, el marco teórico tiene como objetivo abordar la literatura relevante sobre la comunicación de RSC en las redes sociales. La sección 3 discute el corpus y la metodología de la investigación, y la sección 4 comenta los resultados. En la sección final, los resultados son discutidos y comparados con la literatura y se extraen ciertas conclusiones.

\section{ANTECEDENTES DEL ESTUDIO}

\subsection{Comunicación de RSC en España}

Durante las dos últimas décadas, hay una tendencia positiva entre las empresas españolas en lo que respecta a la presentación de informes de RSC. Siete firmas

${ }^{2}$ Esta ley amplía el contenido de la información no financiera sobre la cual las empresas deben informar, como la Responsabilidad Social Corporativa y la sostenibilidad. La ley implica la modificación de varias leyes existentes en el ordenamiento jurídico español y actualmente es una de las más exigentes de toda la Unión Europea. La sección 2.1 proporciona más información sobre esta ley.

Revista de Comunicación de la SEECI. 2021, n 54, 119-143 
Moyaert, H.; Vangehuchten, L y Fernández-Vallejo, A.M. La estrategia de comunicación de RSC de Iberdrola en Facebook y Twitter: Un análisis lingüístico y de contenido basado en

españolas utilizaron el sistema Global Reporting Initiative (GRI) para la elaboración de reportes anuales de RSC en 2002. Este número aumentó a 180 para 2012. Es también importante destacar, que España es el país con mayor número de directrices GRI en implementación (Ministerio de Empleo y Seguridad Social, 2014). En términos de iniciativas públicas y legales, en 2018, se ratificó una nueva ley comercial (Ley 11/2018 de información no financiera y diversidad), que especifica los temas sobre los cuales las empresas deben informar, específicamente en las áreas de medio ambiente, empleados, derechos humanos, corrupción, sociedad, proveedores, consumidores e información fiscal. Por tanto, es seguro asumir que la buena comunicación de RSC en España ahora es exigida por ley, aunque todavía existen algunas dudas sobre el cumplimiento de estos requisitos por parte de las empresas, más concretamente en lo que respecta a la información en las redes sociales, ya que no se proporciona un marco o directrices al respecto. Esto probablemente explica por qué las empresas españolas en general no obtienen una puntuación alta en la información de RSC (Observatorio de Responsabilidad Social Corporativa, 2018).

En cuanto a la calidad de la comunicación española de RSC, el último informe del Observatorio de Responsabilidad Social Corporativa (2018), que fue creado en 2004 para estudiar y promover la RSC, concluye que el nivel de información de RSC en España sigue siendo bastante bajo, con 33,62 puntos de media en una escala de 100. Por motivos de representatividad y relevancia, usaron como muestra las treinta y cinco empresas que a 31 de diciembre de 2018 integraban el índice IBEX 35 de la Bolsa española, entre las que se encuentra Iberdrola. Su objetivo era evaluar la calidad de la información sobre aspectos de Responsabilidad Social Corporativa (RSC) proporcionada en la documentación e informes públicos y accesibles de estas empresas, por lo que estudiaron reportes anuales, reportes de sostenibilidad o reportes similares. Establecieron que si bien todas las empresas analizadas hacen mención explícita en sus informes de sostenibilidad y declaraciones de información no financiera de los problemas y desafíos relacionados con cada una de las áreas de estudio -fundamentalmente en relación con el medio ambiente, la sociedad y la corrupción-, la calidad de la información que presentan respecto a cada uno de estos aspectos aún es deficiente. Además, no alcanza un nivel adecuado en ninguna de las áreas analizadas a pesar de las obligaciones impuestas por la ya mencionada Ley 11/2018, que se utilizó como indicador para el análisis. Sin embargo, cabe mencionar que, al compararlo con la puntuación obtenida en 2017, vemos que la puntuación sube en todas las áreas, lo que significa que la entrada en vigor de la Ley 11/2018 ha tenido el efecto de mejorar la información presentada en esos aspectos de los cuales se ocupa. Además, por sector de actividad, el que obtiene la mejor puntuación es el de Petróleo y Energía. Entre ellos se encuentra Iberdrola, que, si bien puntúa por debajo de los 50 -con 49,66 puntos en una escala de 100-, se sitúa en el tercer puesto de las 35 empresas. Las razones que da el Observatorio de RSC (Observatorio de Responsabilidad Social Corporativa, 2018) sobre por qué el sector de Petróleo y Energía está mejor posicionado, pueden estar relacionadas con una mayor presión social y, por tanto, un mayor grado de vigilancia por parte de la sociedad civil y los medios de comunicación, ya que las empresas realizan actividades con altos riesgos de impacto ambiental y de derechos humanos. Por otro lado, este es un sector de 
Moyaert, H.; Vangehuchten, L y Fernández-Vallejo, A.M. La estrategia de comunicación de RSC de Iberdrola en Facebook y Twitter: Un análisis lingüístico y de contenido basado en corpus

actividad que está sujeto a mayores requerimientos regulatorios en el campo ambiental y tributario, entre otros.

\subsection{Comunicación de RSC en las redes sociales}

Tradicionalmente, una empresa comunica sus esfuerzos de RSC mediante la publicación de reportes, anuncios o sitios web (Du et al., 2010). Ahora, gracias a la Web 2.0, son posibles conversaciones interactivas online a través de una amplia gama de alternativas como blogs y microblogs, comunidades de contenido, sitios de redes sociales y marcadores o agregadores sociales (Coombs y Holladay, 2012). Este altamente exitoso método de marketing lleva a las empresas a repensar sus métodos para comunicar los sistemas de RSC y la incorporación de las redes sociales en su política de comunicación actual se ha vuelto crucial para las empresas (Brown y Nwagbara, 2018).

El estudio de Postma (2015) ofrece evidencia cualitativa de los enfoques de las empresas sobre el uso de las redes sociales cuando se involucran en RSC. Sin embargo, las organizaciones en su estudio no están de acuerdo sobre la idoneidad de las redes sociales para comunicar sobre temas relacionados con la RSC. Esta división es consistente con resultados contradictorios en la literatura (Fieseler et al., 2010; Haigh et al., 2013; Eberle et al., 2013). La mayoría de las empresas abogan por una combinación de medios, debido a la inestabilidad de las redes sociales y la falta de participación de los grupos de interés -que en cambio se puede mejorar a través del diálogo con los grupos de interés-. Postma demuestra la dificultad de aplicar la experticia teórica en acción. La discrepancia entre la teoría de la RSC y la realidad indica que las organizaciones necesitan una guía práctica sobre cómo la teoría realmente debería ponerse en práctica. Un marco sobre el diseño, las aplicaciones y la comunicación de una política de RSC podría proporcionar una solución para cerrar esta brecha (Postma, 2015).

Brown y Nwagbara (2018) también han descrito algunos de los beneficios y desafíos de la comunicación de RSC en las redes sociales. En primer lugar, señalan que las redes sociales ayudan a las empresas a escuchar a las audiencias objetivo y realizar un seguimiento de los problemas particulares relacionados con la RSC que son relevantes para las diferentes partes interesadas. En segundo lugar, las empresas pueden aumentar la legitimidad de su comportamiento de RSC, aunque existe un creciente escepticismo sobre las actividades de RSC que se informan a través de los medios de comunicación (Du et al., 2010). Además de muchas otras ventajas, como inversión barata, rendimiento, comunicación en tiempo real, relaciones públicas y elección de archivos en línea, las redes sociales también tienen varios obstáculos para la comunicación de RSC. Desafortunadamente, no obedecer las reglas de etiqueta, como imponer información no solicitada (Coombs y Holladay, 2012) puede infringir fácilmente un medio tan valioso. Otro problema es el hecho de que las empresas tienen poca o ninguna influencia sobre la información de RSC que fluye a través de las redes sociales, que fácilmente puede volverse negativa y es imposible de predecir (Friedman, 2006). Además, el lenguaje de las redes sociales también debe diseñarse cuidadosamente para reflejar la identidad corporativa

Revista de Comunicación de la SEECI. 2021, nº 54, 119-143 
Moyaert, H.; Vangehuchten, L y Fernández-Vallejo, A.M. La estrategia de comunicación de RSC de Iberdrola en Facebook y Twitter: Un análisis lingüístico y de contenido basado en corpus

adecuada. En este sentido, la literatura se centra en el lenguaje emocional, el lenguaje informal y los elementos multimodales.

\subsubsection{Lenguage emocional}

En la Web 2.0, el marketing emocional encuentra una plataforma ideal para crecer. El contacto corporativo convencional, que se caracteriza por la unidireccionalidad y verticalidad, se ha enriquecido y ha surgido una forma moderna de comunicarse, implicando una comunicación multidireccional en la que participan todos los grupos de interés de la organización. También el uso de redes, en particular Facebook, Twitter, Instagram y LinkedIn, ha experimentado un enorme crecimiento en el clima organizacional (Han \& Chang-Hoan, 2013).

Hasta ahora, la investigación que combina emociones y sentimientos en Twitter se ha centrado principalmente en "marketing" y problemas sociológicos, pero no en cuestiones lingüísticas. En los últimos años, se han realizado algunos esfuerzos para encontrar mecanismos analíticos capaces de examinar qué tipo de emociones están presentes tanto en los tweets como en otras formas de publicaciones en redes y cómo estas emociones afectan la 'viralidad' (Stieglitz, 2013). Sin embargo, son escasos los estudios que examinan las emociones en Twitter en el contexto empresarial español desde un punto de vista lingüístico, a excepción de FernándezVallejo (2018). Realizó un estudio en el que se investiga la expresión de las emociones en las cuentas de Twitter corporativas españolas (incluida Iberdrola) para observar qué estructuras lingüísticas caracterizan o provocan emociones, y qué sistemas léxicos conceptualizan y valoran el campo semántico de las emociones. Su estudio confirmó la validez de los mensajes corporativos como corpus relevante para el estudio de la expresión de las emociones en el entorno organizacional, dado el alto porcentaje de tuits que describen o provocan emociones. La investigación indicó que las que predominan en los tuits de Acciona e Iberdrola fueron las emociones de valencia positiva como la alegría, la seguridad y la confianza, que genera respeto y lealtad en el accionista o consumidor. Claramente, las emociones suscitadas basadas en el propósito, la visión y los valores de la empresa ayudan a reforzar la identidad de la organización.

\subsubsection{Lenguaje informal}

Sin duda, Internet ha revitalizado el secular debate sobre las características de lo oral y las características de lo escrito, y ha mencionado también la grafología distintiva que tiene, que se basa en la singularidad y creatividad léxico-gráfica de los mensajes en Internet (Crystal y Tena, 2002). Si bien las redes sociales se han convertido en los principales canales de comunicación tanto para los clientes como para las marcas, los especialistas en marketing están luchando en estos canales para establecer vínculos sostenibles entre el consumidor y la marca. Investigaciones recientes muestran que los intentos de los anunciantes de cultivar los lazos de las redes sociales con sus clientes están lejos de ser exitosos (Fournier \& Avery, 2011), donde los consumidores a menudo rechazan los anuncios de marca y critican a las 
Moyaert, H.; Vangehuchten, L y Fernández-Vallejo, A.M. La estrategia de comunicación de RSC de Iberdrola en Facebook y Twitter: Un análisis lingüístico y de contenido basado en corpus

marcas en sus espacios sociales (Van Noort \& Willemsen, 2011; Gretry, Horváth, Belei y van Riel, 2017).

En estos casos, las señales no verbales, como el estilo de contacto, juegan un papel fundamental en la construcción de la confianza $y$, posteriormente, en la determinación de sí la relación avanzará más allá de los encuentros iniciales (Keeling McGoldrick y Beatty, 2010). Para investigar esto, Gretry et al. (2017) llevaron a cabo un estudio en el que examinaron cómo el uso de un estilo de comunicación informal (vs. formal) afecta la confianza de la marca en un contexto de redes sociales. Los experimentos muestran que los efectos de un estilo informal sobre la confianza de la marca dependen de si los consumidores están familiarizados con la marca, de modo que el uso de un estilo informal mejora (disminuye) la confianza en las marcas con las que los consumidores están familiarizados (no familiarizados). En particular, las marcas parecen utilizar un estilo predominantemente informal en sus comunicaciones en las redes sociales (Beukeboom, Kerkhof \& de Vries 2015), ya que creen que fomenta la cercanía. Un estilo de comunicación informal se define como "común, no oficial, familiar, casual y, a menudo, coloquial, y contrasta con lo formal en ese sentido" (McArthur, 1992). Por ejemplo, es común que los especialistas en marketing se refieran a sus clientes por su nombre y utilicen emoticonos $y / 0$ expresiones abreviadas. También se observa libertad en el uso de mayúsculas y minúsculas, la revitalización de signos de puntuación especiales, o el endulzamiento de las reglas académicas de puntuación (Crystal \& Tena, 2002; Fernández-Vallejo, 2020).

\subsubsection{Elementos multimodales}

Los elementos multimodales también son un aspecto esencial del discurso de las redes sociales, ya que influyen en la capacidad de búsqueda. El hashtag, un tipo de etiquetado social que permite a los microblogueros inyectar metadatos en las publicaciones de las redes sociales, es una herramienta semiótica clave que respalda esta función. Aunque popularmente se los considera marcadores de temas, los hashtags en los textos de las redes sociales pueden interpretar una variedad de significados complejos. Anotar publicaciones en redes sociales con estas etiquetas es una actividad de ritmo rápido, pero en evolución y un proceso de innovación lingüística. Según lo establecido por Zappavigna (2015), los hashtags pueden servir para múltiples cosas. Pueden servir como marcadores de temas -su posición más comúnmente reconocida-, pero también pueden construir significados interpersonales o promulgar metacomentarios a nivel de discurso semántico. Además, pueden verse como una forma de etiquetado social, porque la anotación de texto sirve como concepto de "búsqueda" en la red social.

Además de los hashtags, las etiquetas o hipervínculos apelativos también pueden ser considerados como un tipo especial de estrategia inherente al discurso de las redes sociales, que consiste en referenciar el perfil de audiencia relevante para incrementar los cambios de un comentario (Vela Delfa, 2017). Aunque no se puede encontrar mucha literatura sobre teorías sobre el uso de esto para el discurso de las redes sociales, De Choudhury, Sundaram, Lin, John \& Seligmann (2009), concluyeron que el 
Moyaert, H.; Vangehuchten, L y Fernández-Vallejo, A.M. La estrategia de comunicación de RSC de Iberdrola en Facebook y Twitter: Un análisis lingüístico y de contenido basado en corpus

uso de hipervínculos apelativos mejora significativamente el desempeño de las recomendaciones frente al contenido de imagen sola.

\section{METODOLOGÍA}

\subsection{Selección de corpus.}

Para responder a las preguntas de investigación planteadas en la introducción, se examinaron el contenido y los elementos textuales de un corpus de 438 Tweets y 126 publicaciones de Facebook publicadas por la firma española Iberdrola (ver tabla 1). Iberdrola fue elegida como firma por formar parte del IBEX 35, índice bursátil de referencia de la Bolsa de Madrid, la principal bolsa de valores de España. En este caso concreto, Iberdrola es uno de los líderes del sector de Petróleo y Energía. Además, tiene un fuerte compromiso con la sostenibilidad y la RSC a nivel ambiental y cultural, lo que los ha llevado a desarrollar patrocinios de iniciativas sociales. De hecho, según la $15^{a}$ edición del 'Global 100 Most Sustainable Corporations in the World', que publica anualmente Corporate Knights, Iberdrola fue nombrada como una de las 100 empresas más sostenibles del mundo.

Otro elemento importante de por qué se eligió Iberdrola, es el hecho de que ocupa un lugar destacado en la gestión de sus redes sociales, destacando por fans, interacciones, viralidad y engagement (según un estudio realizado por Epsilon en 2016). Además de eso, tienen una buena cantidad de seguidores, lo que es relevante en cuanto a las razones por las que mantienen tal lealtad de los grupos de interés en las redes sociales. El número actual de seguidores en Twitter es 65.652 y en Facebook es 76.242. Esto es bastante interesante, porque cuando Fernández-Vallejo (2018) realizó un estudio sobre la presencia online de Iberdrola, el número de seguidores en Twitter era de solo 48.055 .

El corpus cubre un período de tres meses desde el 1 de octubre de 2019 hasta el 31 de diciembre de 2019. Este período estuvo marcado por algunas fiestas importantes, como Navidad y Nochevieja, y un gran evento en Madrid, a saber, la COP25. La Conferencia de las Naciones Unidas sobre el Cambio Climático de 2019 fue la $25^{\circ}$ conferencia de las Naciones Unidas sobre el Cambio Climático y se celebró en Madrid del 2 al 13 de diciembre. Sin duda, esto ha tenido un gran impacto en la estrategia de redes sociales de Iberdrola. Otras observaciones importantes fueron el patrocinio de varios eventos, la implementación de obras de infraestructura relacionadas con la sostenibilidad y las iniciativas de RSC, pero estas ocurren durante todo el año.

Para la obtención del corpus se utilizó el programa Twlets [http://twdocs.com] para Twitter, que descargó directamente los tweets en una hoja de Excel. Para Facebook, el corpus se obtuvo manualmente y se colocó en un documento de Word. Se aplicó una prueba previa a 30 tweets y 30 publicaciones de Facebook para verificar si los temas seleccionados (que se seleccionaron a través de una consulta de frecuencia de palabras) eran aplicables a la muestra. 
Moyaert, H.; Vangehuchten, L y Fernández-Vallejo, A.M. La estrategia de comunicación de RSC de Iberdrola en Facebook y Twitter: Un análisis lingüístico y de contenido basado en corpus

En resumen, el corpus aborda el estudio empírico a pequeña escala, convencido con Thornbury (YEAR) de que un corpus pequeño y homogéneo también puede ser válido. La aproximación al estudio del lenguaje se adopta desde dos perspectivas complementarias, el análisis de contenido y lingüístico.

Tabla 1. Número de Tweets y publicaciones de Facebook analizadas.

\begin{tabular}{|c|c|c|c|}
\hline Corpus & $\begin{array}{c}\text { Número de } \\
\text { publicaciones }\end{array}$ & $\begin{array}{c}\text { Palabras } \\
\text { analizadas }\end{array}$ & $\begin{array}{c}\text { Promedio de } \\
\text { publicaciones } \\
\text { por día }\end{array}$ \\
\hline Twitter & 438 & 12.759 & 4,76 \\
\hline Facebook & 126 & 5.313 & 1,36 \\
\hline Total & 564 & 18.072 & \\
\hline
\end{tabular}

\subsection{Técnicas de investigación.}

El objetivo de este estudio es examinar la comunicación de RSC de una empresa de habla hispana, en este caso Iberdrola, en Facebook y Twitter. Twitter y Facebook se consideran dos partes separadas de la estrategia de comunicación digital, que se examina en términos de contenido y potencial interactivo.

El análisis de contenido se estructuró de la siguiente manera. En primer lugar, se llevó a cabo la parte deductiva, es decir, la primera parte del estudio se dedicó a investigar la literatura previa sobre RSC y redes sociales. Rápidamente se hizo evidente que, aunque existen muchos marcos para la comunicación de RSC en los informes (como las pautas de GRI), no había muchos marcos en torno a la comunicación de RSC en las redes sociales, ni había claridad sobre su idoneidad. Existe mucha discrepancia entre la teoría de la RSC y la realidad y, como se indicó en la sección anterior, las organizaciones no están seguras de la idoneidad de las redes sociales para la RSC, a menudo abogando por una combinación de medios (Postma, 2015). También existe confusión sobre el impacto de la comunicación de RSC en las redes sociales, con opiniones encontradas (Cho, Furey \& Mohr, 2016; Etter, 2013). Aquí es donde surge la necesidad de desarrollar estrategias de comunicación de RSC para alentar al público a involucrarse con las corporaciones, lo que demuestra varios aspectos de la RSC al incluir tanto a los públicos internos como externos involucrados en los programas (Cho et al., 2016).

En segundo lugar, se llevó a cabo una consulta de frecuencia de palabras en Nvivo $^{3}$, que tiene como objetivo identificar posibles temas, particularmente en las primeras etapas de un proyecto. El resultado de este enfoque inductivo se combinó con el enfoque deductivo y luego se utilizó para codificar el contenido de todas las publicaciones. Para verificar la confiabilidad del método, también se le pidió a un segundo codificador que evaluara el $10 \%$ de los Tweets y las publicaciones de Facebook (44 Tweets y 13 publicaciones de Facebook). Los porcentajes de

\footnotetext{
3 NVivo es una aplicación de software que se utiliza para el análisis utilizando enfoques tanto cualitativos como mixtos. Universidad Estatal de Kent. (s.f.).
}

Revista de Comunicación de la SEECI. 2021, nº 54, 119-143 
concordancia entre los dos codificadores que realizaron la tarea fueron superiores al $89 \%$ para todos los temas específicos del contenido, e incluso mayores para los temas textuales. Para tener en cuenta la probabilidad de que el acuerdo entre usuarios se produzca por casualidad, también se midió el coeficiente Kappa. El coeficiente Kappa siempre estuvo entre $0,55(k>0)$ y $1,00(k=1)$ para Twitter, que es una concordancia entre regular y muy buena, y entre $0,33(k>0)$ y $1,00(k=1)$ para Facebook.

En cuanto a la parte lingüística del análisis, todas las publicaciones fueron codificadas en base a la presencia de elementos emocionales, informales y multimodales, como se expone en la sección 2. La siguiente sección presenta los resultados del análisis de contenido y el lingüístico.

\section{RESULTADOS}

\subsection{Análisis de contenido}

El contenido de los Tweets y las publicaciones de Facebook se analizó de acuerdo a 8 temas, que se pueden encontrar en la tabla siguiente (ver tabla 2). Debido al hecho de que las publicaciones a menudo trataban más de un tema y, por lo tanto, se codificaron varias veces, muchos temas tienen un alto número de referencias codificadas Los resultados del contenido de RSC serán discutidos de acuerdo a la frecuencia y/u ocurrencia de los temas.

Tabla 2. Resultados del análisis de contenido

\begin{tabular}{|c|c|c|c|c|}
\hline \multirow{2}{*}{ Temas } & \multicolumn{2}{|c|}{ Twitter } & \multicolumn{2}{c|}{ Facebook } \\
\cline { 2 - 5 } & $\begin{array}{c}\text { Frecuencia } \\
\text { de } \\
\text { referencias } \\
\text { codificadas }\end{array}$ & $\begin{array}{c}\text { Porcentaje } \\
\text { de cobertura }\end{array}$ & $\begin{array}{c}\text { Frecuencia } \\
\text { de } \\
\text { referencias } \\
\text { codificadas }\end{array}$ & $\begin{array}{c}\text { Porcentaje } \\
\text { de } \\
\text { cobertura }\end{array}$ \\
\hline Tema ambiental & 120 & $13.70 \%$ & 22 & $13.89 \%$ \\
\hline Sustentabilidad & 129 & $14.73 \%$ & 14 & $9.16 \%$ \\
\hline Intereses y asuntos de actualidad & 148 & $16.88 \%$ & 69 & $47.03 \%$ \\
\hline Partes interesadas & 148 & $16.88 \%$ & 44 & $31.49 \%$ \\
\hline Proyectos & 92 & $10.50 \%$ & 29 & $19.25 \%$ \\
\hline Referencias culturales & 39 & $4.45 \%$ & 15 & $12.82 \%$ \\
\hline Innovación & 21 & $2.40 \%$ & 1 & $0.60 \%$ \\
\hline Concursos & 53 & $6.05 \%$ & 48 & $42.55 \%$ \\
\hline
\end{tabular}

Tanto para Twitter como para Facebook, "Intereses y asuntos de actualidad" tiene el mayor porcentaje de cobertura. Este tema se codificó cuando los Tweets o publicaciones de Facebook eran sobre deportes, días específicos/festividades, eventos $y$, en menor cantidad, animales. Consulte las publicaciones a continuación para ver un ejemplo de festividades (1) y animales (2). 
Moyaert, H.; Vangehuchten, L y Fernández-Vallejo, A.M. La estrategia de comunicación de RSC de Iberdrola en Facebook y Twitter: Un análisis lingüístico y de contenido basado en corpus

1. Primer ítem "\#Halloween da mucho MIEDO por un día, pero destruir nuestro nos debe alarmar SIEMPRE iNo convirtamos el planeta en un escenario de TERROR! \#halloween2019 \#LordPlastik"

2. "Cuidar a los animales en la naturaleza y en casa nos define como seres humanos. iProtejámoslos! \#DíaMundialdelosAnimales"

Lo que es distinto es que para Twitter, el tema "Partes interesadas" también ocupa el primer lugar (148 referencias codificadas tanto para "Partes interesadas" como para "Intereses y asuntos de actualidad"). Las "Partes interesadas" se codificaron de acuerdo con los subtemas de clientes, empleados, mujeres y familias. A continuación se puede encontrar un ejemplo de clientes (3) y mujeres (4).

3. "En \#Navidad y SIEMPRE, nos comprometemos a NO CORTAR la luz a nuestros clientes en situación vulnerable que no puedan pagar sus facturas, gracias a acuerdos con instituciones y ONG"

4. "¿A qué retos se enfrentan las mujeres en el nuevo entorno digital? Expertas del sector piden más igualdad de oportunidades en el I Encuentro de mujeres digitales \#EmakumeDigitalak en \#Bilbao 숩

Para el segundo tema más concurrente, existe una diferencia notable entre la comunicación en Twitter y Facebook. En Twitter, el segundo tema más común es "Sostenibilidad", con tres subtemas; energías renovables, reducción de emisiones y productos/servicios (por el hecho de que el negocio de Iberdrola se enmarca en el sector de las energías sostenibles). Un ejemplo de energía renovable (5) es el siguiente:

5. "Terminó la \#COP25, pero el compromiso y ACCIÓN por el clima CONTINÚA $\rightarrow$ Impulsamos las \#renovables y la sostenibilidad por un mejor para TODOS \#TiempoDeActuar"

Esto implica una gran diferencia con la estrategia de comunicación en Facebook, donde los "Concursos" (aunque sean para motivar a los stakeholders y relacionados con la RSC) son el segundo tema principal. Mientras que los concursos ocupan el segundo lugar en Facebook, solo ocupan el quinto lugar en Twitter. Lo mismo ocurre con "Sostenibilidad", que solo ocupa el sexto lugar en Facebook. Esto significa que ambos canales otorgan un valor diferente a estos temas. Un ejemplo de una publicación de Facebook con un tema de concurso (6) es el siguiente:

6. "Frenar el cambio climático es imprescindible para dejar un habitable a los que vienen... iy a nosotros! Reivindícalo en Instagram y participa en nuestro concurso \#PorelClima junto a @IgersSpain, con motivo de la \#COP25 en Madrid."

El tercer tema más frecuente para Twitter es el "tema medioambiental", mientras que para Facebook es "Stakeholders", y después "Proyectos" (social, cultural, medioambiental, etc.). Estos son intercambiables para el siguiente tema, con "Proyectos" ocupando este espacio en Twitter y "Temas medioambientales" ocupando este espacio en Facebook. Esto significa que tanto el tema ambiental como 
el social tienen aproximadamente la misma importancia en ambas redes sociales, con casi siempre más del $13 \%$ de porcentaje de cobertura. El tema ambiental se codificó de acuerdo con tres subtemas; cuestiones medioambientales, respeto/valoración del medio ambiente, consejos medioambientales. A continuación, se puede encontrar un ejemplo de proyectos (7) y cuestiones medioambientales (8).

7. "Pippi Calzaslargas tenía a Pequeño Tío, su "gran" ayudante y amigo 2 ... y en el mundo real, niños con discapacidad psíquica tienen a los de la Fundación @Caballo_Amigo, con la que colaboramos en 睇, para ayudarles a desarrollar sus habilidades e integración (:)"

8. "NO, no es algo NATURAL. Ni obra de un agente secreto . No lo trajeron los extraterrestres(-). No llegó ahí por casualidad 0 . La culpa de la INVASIÓN del plástico es nuestra. Hagamos caso a Cousteau, el gran defensor del @, y paremos YA a \#LordPlastik 四 $Q$ "

Los temas que están presentes en menor medida, aunque deben mencionarse, son "Referencias culturales" (9) e "Innovación". Más determinante, la "Innovación" no puede ser considerada como parte de la comunicación de RSC para Facebook, mientras que el tema sí estuvo presente en Twitter. Esto podría deberse al hecho de que la comunicación de Twitter es más RSC en su forma pura, que es informar, y en Facebook casi siempre es una combinación de informar e interactuar.

9. "El pavo, el marisco, el jamón, el pescado, la ensalada, el cava, el turrón, el (3...En \#Navidad y SIEMPRE, necesitamos a la gente de campo y mar. Sin nuestros pueblos, no tendríamos NADA. iPorque no solo lucen las ciudades!

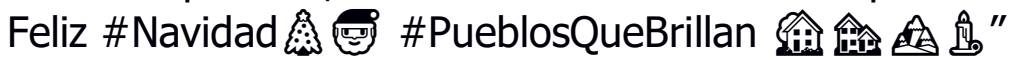

\subsection{Análisis lingüístico}

Los resultados del discurso lingüístico tanto de los Tweets como de las publicaciones de Facebook se discutirán de acuerdo con la frecuencia/ocurrencia de los elementos textuales, como se presenta en la tabla siguiente (ver tabla 3).

Tabla 3. Resultados del análisis lingüístico

\begin{tabular}{|c|c|c|c|c|c|}
\hline \multicolumn{2}{|c|}{ Temas } & \multicolumn{2}{|c|}{ Twitter } & \multicolumn{2}{c|}{ Facebook } \\
\cline { 3 - 6 } & $\begin{array}{c}\text { Frecuencia } \\
\text { de } \\
\text { referencias } \\
\text { codificadas }\end{array}$ & $\begin{array}{c}\text { Porcentaje } \\
\text { de } \\
\text { cobertura }\end{array}$ & $\begin{array}{c}\text { Frecuencia } \\
\text { de } \\
\text { referencias } \\
\text { codificadas }\end{array}$ & $\begin{array}{c}\text { Porcentaje } \\
\text { de } \\
\text { cobertura }\end{array}$ \\
\hline $\begin{array}{c}\text { Emociones } \\
\text { Sustentabilidad }\end{array}$ & $\begin{array}{c}\text { Valencia } \\
\text { negativa }\end{array}$ & 69 & $7.88 \%$ & 18 & $12.06 \%$ \\
\cline { 2 - 6 } & $\begin{array}{c}\text { Valencia } \\
\text { positiva }\end{array}$ & 312 & $35.60 \%$ & 100 & $72.11 \%$ \\
\hline $\begin{array}{c}\text { Elementos textuales } \\
\text { informales }\end{array}$ & $\begin{array}{c}\text { Abuso de } \\
\text { signos de } \\
\text { puntuación }\end{array}$ & 155 & $17.69 \%$ & 80 & $57.27 \%$ \\
\cline { 2 - 6 } & Emojis & 388 & $44.28 \%$ & 118 & $86.61 \%$ \\
\cline { 2 - 6 } & $\begin{array}{c}\text { Uso libre de } \\
\text { mayúsculas }\end{array}$ & 248 & $28.30 \%$ & 90 & $62.20 \%$ \\
\hline
\end{tabular}


Moyaert, H.; Vangehuchten, L y Fernández-Vallejo, A.M. La estrategia de comunicación de RSC de Iberdrola en Facebook y Twitter: Un análisis lingüístico y de contenido basado en corpus

\begin{tabular}{|c|c|c|c|c|c|}
\hline & $\begin{array}{c}\text { Mimetismo de } \\
\text { sonido }\end{array}$ & 1 & $0.11 \%$ & $/$ & $/$ \\
\cline { 2 - 6 } & $\begin{array}{c}\text { Uso de } \\
\text { primera y } \\
\text { segunda } \\
\text { persona }\end{array}$ & 308 & $35.15 \%$ & 94 & $73.08 \%$ \\
\hline Elementos multimodales & Hashtags & 329 & $37.56 \%$ & 108 & $81.01 \%$ \\
\cline { 2 - 6 } & Etiquetas & 114 & $13.01 \%$ & 20 & $16.22 \%$ \\
\hline
\end{tabular}

\subsubsection{Emociones}

Tanto Twitter como Facebook tienen más referencias codificadas para valencia positiva (10), aunque Facebook tiene más cobertura porcentual, lo que significa que la valencia positiva general en Facebook es más grande -o más intensa- que en Twitter. La valencia positiva se codificó cuando las publicaciones implicaban un sentimiento de sorpresa, felicidad, confianza u orgullo. Lo mismo ocurre con la valencia negativa (11), que es -aunque se usa menos- más intensa en Facebook. La valencia negativa se codificó cuando estaba implícito el sentimiento de ira, tristeza o miedo. Esto podría significar que la estrategia de comunicación general en Facebook se centra en emociones más fuertes, ya sean positivas o negativas. En Twitter, una pequeña cantidad de publicaciones es simplemente neutral.

10. "¿Y si sonreímos? Usemos bien el mejor recurso: iTU energía positiva, la de TODOS! Construir, amar, disfrutar... :) \#DíaMundialdelasonrisa"

11."留留留留 Cinco islas ENORMES que no figuran en los mapas... Grandes masas de plástico flotante que amenazan a nuestros océanos. iFrenemos YA el plástico de un solo uso! \#TiempoDeActuar \#COP25"

\subsubsection{Elementos textuales informales}

Los emojis constituyen una gran parte de la comunicación (informal) tanto para Twitter como para Facebook, con la cobertura porcentual más alta para ambas redes sociales. El segundo elemento informal más común es el uso de la primera y segunda persona, ya sea el uso de verbos, adjetivos o pronombres personales. Esto cuenta, tanto para Twitter, como para Facebook.

El uso libre de mayúsculas ocupa el tercer lugar tanto para Twitter como para Facebook. Esto significa que muchas palabras se escriben completas con letras mayúsculas cuando el énfasis está en esa palabra. Esto es muy inusual e informal viniendo de una gran corporación.

El abuso de signos de puntuación está presente en menor medida, aunque no es menos importante que los otros elementos informales. Aunque se han codificado menos referencias, el porcentaje de cobertura es bastante alto, por lo que tienen una gran importancia a la hora de la comunicación en redes sociales. Sin embargo, la imitación de sonido tiene poca o ninguna influencia, con solo una referencia codificada.

\subsubsection{Elementos multimodales}

Revista de Comunicación de la SEECI. 2021, n 54, 119-143 
Moyaert, H.; Vangehuchten, L y Fernández-Vallejo, A.M. La estrategia de comunicación de RSC de Iberdrola en Facebook y Twitter: Un análisis lingüístico y de contenido basado en corpus

En ambas redes sociales, Twitter y Facebook, los hashtags juegan un papel importante. Con un porcentaje de cobertura de alto a muy alto, 37,56\% para Twitter y $81,01 \%$ para Facebook, es seguro asumir que son muy recurrentes. Sin embargo, es relevante mencionar que el porcentaje para Facebook es mucho mayor. Como se dijo anteriormente, esto puede deberse a que la estrategia comunicativa en Facebook es más intensa, por lo que se espera una mayor interactividad de los grupos de interés. Twitter, por otro lado, también puede ser puramente informativo (para RSC), y no solo se centra en la interactividad.

En términos de etiquetas de otras personas o corporaciones, Facebook y Twitter tienen aproximadamente la misma cantidad de cobertura, es decir, no hay gran diferencia en términos de comunicación de RSC. El porcentaje de cobertura también es alto, que se sitúa entre el $13 \%$ y el $16 \%$.

\subsection{Integración del análisis de contenido y lingüístico}

A continuación, se discutirán las correlaciones entre los hallazgos en el análisis de contenido y los hallazgos en el análisis lingüístico, para revelar cualquier patrón que pueda estar presente. El propósito de esta sección es responder una serie de preguntas que pueden surgir cuando se muestran patrones en los datos.

Tanto para Twitter como para Facebook, el tema con la valencia más positiva es el tema "Partes interesadas", lo que significa que la valencia positiva se usa principalmente cuando las publicaciones están dirigidas a las partes interesadas. Para Twitter, la valencia negativa se usa principalmente cuando se informa sobre temas y problemas ambientales. Sin embargo, para Facebook, el tema con mayor porcentaje de valencia negativa es "Intereses y asuntos de actualidad". No obstante, está muy cerca a "Temas medioambientales", con solo una referencia codificada menos. Por tanto, se puede suponer que no existe una gran diferencia en el tipo de valencia entre Facebook y Twitter.

Otra observación llamativa son los diferentes tipos de elementos textuales informales que están vinculados a tipos de contenido, que también varían según la red social. En Twitter, el tema "Stakeholders", por ejemplo, se combina principalmente con "Emojis", aunque debe tenerse en cuenta que los "Emojis" tienen un alto porcentaje de cobertura general. "Intereses y asuntos de actualidad" se combinan muy a menudo con "Abusivo de signos de puntuación", "Uso libre de mayúsculas" y "Uso de $1^{\mathrm{a}}$ y $2^{\mathrm{a}}$ persona". Esto probablemente se deba al hecho de que estos temas implican a las partes interesadas más que otros temas $\mathrm{y}$, por lo tanto, interactúan mucho en términos de estrategia.

Sin embargo, con respecto a los elementos textuales informales para Facebook, todos los elementos textuales se utilizan predominantemente cuando se comunica sobre "Intereses y asuntos de actualidad". Además, el segundo tema con el que se combinan mayoritariamente todos los elementos textuales es "Concursos". Esto es bastante diferente a Twitter, donde también se usaban con frecuencia 
Moyaert, H.; Vangehuchten, L y Fernández-Vallejo, A.M. La estrategia de comunicación de RSC de Iberdrola en Facebook y Twitter: Un análisis lingüístico y de contenido basado en corpus

"Sostenibilidad" y "Temas medioambientales". Como se mencionó anteriormente, esto podría deberse a que se espera una mayor interactividad de los interesados en Facebook y, por lo tanto, la atención se centra en otros temas.

En cuanto a los elementos multimodales, tanto para Twitter como para Facebook, los hashtags son los más utilizados a la hora de comunicar sobre "Intereses y asuntos de actualidad", probablemente por el hecho de que Iberdrola suele utilizar como ventaja eventos diarios, como festividades y eventos deportivos. Sin embargo, es importante señalar que estos eventos diarios casi siempre están vinculados a una actividad o tema de RSC. Las etiquetas se utilizaron con mayor frecuencia cuando se comunicaba sobre "Concursos", para motivar a las partes interesadas y aumentar aún más la interactividad, pero también para informarles sobre proyectos y asociaciones con las que están trabajando.

\section{DISCUSIÓN}

Los resultados del apartado anterior muestran que Iberdrola combina una estrategia de contenidos informativos y una estrategia lingüística interactiva en su comunicación de RSC en redes sociales, dando lugar a interesantes observaciones, que contribuyen a un marco más claro para la comunicación de RSC en redes sociales en general.

\subsection{Estrategia de información de Iberdrola en redes sociales.}

Si miramos los resultados de la frecuencia de los temas de RSC, hay ciertas conclusiones que podemos sacar para la estrategia de información de Iberdrola. En general, las publicaciones que involucraron deportes, festividades específicas y/o días temáticos, eventos y animales estuvieron presentes con mayor frecuencia, tanto en Twitter como en Facebook. Sin embargo, estos temas casi nunca estuvieron presentes solos, por lo que siempre estuvieron acompañados de un tema de RSC más serio, como un tema ambiental, un tema de sostenibilidad, etc. Esto significa que la estrategia de información de Iberdrola consiste en combinar temas serios de RSC con asuntos como intereses generales y asuntos de actualidad. Lo más probable es que esto sirva para aumentar el interés y el compromiso de las partes interesadas (menos conscientes), con el fin de comunicar los puntos de la empresa sobre RSC de una manera más accesible.

Lo mismo ocurre con el tema "Partes interesadas", que ocupa una posición bastante alta en ambos canales de redes sociales. Este tema se codificó de nuevo a menudo con otros temas serios de RSC, pero en algunos casos también los suyos. Esto podría explicarse por el hecho de que nuevamente se trata de una estrategia que sirve para incrementar el compromiso de los grupos de interés, sean o no clientes existentes, hacia temas relacionados con la RSC. Sin embargo, este tema también se puede considerar como un tema de RSC "más puro", porque no solo se dirige a las familias y los clientes actuales o futuros, sino también a las mujeres y los empleados. Por lo tanto, tiene un doble efecto, porque se trata de hablar sobre la 
Moyaert, H.; Vangehuchten, L y Fernández-Vallejo, A.M. La estrategia de comunicación de RSC de Iberdrola en Facebook y Twitter: Un análisis lingüístico y de contenido basado en corpus

integridad y los derechos humanos, así como de involucrar e influir en los clientes potenciales.

En cuanto al siguiente tema principal, hay una diferencia notable entre la comunicación en Twitter y la comunicación en Facebook. En Twitter, el segundo tema de RSC más común es sobre sostenibilidad, mientras que en Facebook, los concursos (aunque sean para motivar a las partes interesadas y relacionados con la RSC) se codifican con mayor frecuencia. Por tanto, podemos suponer que la comunicación de Iberdrola varía entre Twitter y Facebook, probablemente porque quieren dirigirse a diferentes tipos de grupos de interés. Si bien Twitter sirve para contenido de RSC "más puro", Facebook trata de dirigirse a las partes interesadas y aumentar la participación, ya sea por razones de marketing o de RSC. Esto puede explicarse por el hecho de que el contenido de Twitter es más difícil de "difundir" que el contenido de Facebook y, por lo tanto, está dirigido a personas que ya están interesadas en el contenido de Iberdrola y ya siguen la cuenta. Facebook, por otro lado, funciona como una forma de motivar a las partes interesadas a aumentar su interés en la información de RSC.

A partir de entonces, los temas y proyectos ambientales se codificaron con mayor frecuencia, teniendo aproximadamente la misma importancia en ambas redes sociales. Son temas puramente relacionados con la RSC, y ambos tienen porcentajes de cobertura elevados, casi siempre superiores al $13 \%$. Por lo tanto, se puede concluir que aunque los temas "atractivos" tienen una importancia considerable, los temas más puros relacionados con la RSC ciertamente tienen mucho peso también. Es importante señalar que, si bien Iberdrola es una empresa energética y, por lo tanto, se espera que comunique más sobre la reducción de emisiones y tal, los temas sociales no son de ninguna manera inferiores a los temas ambientales y renovables. Es decir, si bien se espera que Iberdrola prefiera ciertos temas, la firma ciertamente no. En cierto modo, esto podría reducir los posibles prejuicios de las partes interesadas sobre el "greenwashing", simplemente porque Iberdrola comunica sobre mucho más.

Los temas que están presentes en menor medida, aunque deben mencionarse, son los referentes culturales, es decir, el arte y el patrimonio local y la innovación. Ambos están presentes en Twitter en mayor medida, por lo que podemos suponer que la estrategia de comunicación en Twitter es más sobre RSC en su forma pura, que es informar, mientras que Facebook combina mucho más la información y la participación.

Como se menciona en el marco teórico, el Observatorio de RSC (2018) concluyó que la calidad de la información de RSC en España en 2018 era todavía bastante baja, y que la información presentada por la mayoría de las empresas estaba muy alejada del objetivo de rendición de cuentas perseguido por estándares como la Ley $11 / 2018$, ya que estaba más orientada a la presentación de logros empresariales y buenas prácticas. Sin embargo, el sector que mejor puntuación ha obtenido ha sido el de Petróleo y Energía, entre los que se encuentra Iberdrola, como tercera de las 35 empresas. Si nos fijamos en la comunicación de RSC de Iberdrola en las redes

Revista de Comunicación de la SEECI. 2021, nº 54, 119-143 
Moyaert, H.; Vangehuchten, L y Fernández-Vallejo, A.M. La estrategia de comunicación de RSC de Iberdrola en Facebook y Twitter: Un análisis lingüístico y de contenido basado en corpus

sociales, podemos concluir que, en general, está en línea con sus informes de RSC, con muchas publicaciones relacionadas con los derechos humanos y la transparencia con los empleados. Sin embargo, no hubo un solo post relacionado con la corrupción, algo que posiblemente sea un tabú en España. Por supuesto, no está seguro si mencionar esto mejoraría, o más bien empeoraría, la participación de las partes interesadas.

Además, si comparamos el presente estudio con hallazgos anteriores sobre el sector energético (De Rosario et al., 2011), podemos concluir que Iberdrola sí comunica sobre desempeño ambiental y desarrollo social, pero no sobre valor económico, lo que indica que los accionistas ciertamente no son el primer grupo objetivo en las estrategias de redes sociales de las empresas de petróleo y energía. Asimismo, en la comunicación de Iberdrola sobre preocupaciones medioambientales, la compañía no teme comunicar sobre las emisiones de $\mathrm{CO} 2$, que es un impacto negativo de las empresas de Petróleo y Energía. Esto parece ser lo contrario al estudio de Van Zandvoort (2016), aunque es importante mencionar que Iberdrola ya lo hace bien en términos de energía sostenible, por lo que tienen aspectos más positivos sobre los que comunicar.

\subsection{Estrategia interactiva de Iberdrola en redes sociales.}

Las emociones que predominan en este estudio son las de valencia positiva, que en su mayoría están codificadas con el tema "Stakeholders". Esto confirma la observación de Fernández-Vallejo $(2018,2020)$ de que la estrategia comunicativa en las redes sociales se trata de generar contenido agradable para los grupos de interés específicamente. Lo mismo ocurre con Facebook, con un porcentaje de cobertura aún mayor para las publicaciones con valencia positiva.

Dado que el uso de emociones positivas tiene como objetivo generar admiración y confiabilidad, y reforzar la identidad de la organización, se puede considerar como parte de la estrategia interactiva de las redes sociales. Como se mencionó anteriormente, Iberdrola tiene altas calificaciones en términos de compromiso y la gestión de sus redes, lo que significa que provocar emociones positivas sí ayuda. Las únicas pocas estructuras que provocaron emociones negativas fueron acompañadas de contenido ambiental, lo que significa que una combinación sutil de ambas puede ser favorable para evitar indicios de greenwashing.

En cuanto a los elementos textuales informales, se codificaron con mucha frecuencia, lo que significa que casi toda la estrategia de redes sociales de Iberdrola se basa en un lenguaje informal. Si bien el estudio de Gretry et al. (2017) afirma que un estilo informal puede percibirse como inapropiado, Iberdrola lo aprovecha como una ventaja, y ciertamente puede considerarse como parte de la estrategia interactiva. Sin embargo, cabe señalar que el estudio de Gretry et al. se basó en contenido de marketing y no en contenido de RSC.

En cierto modo, esto significa que el principal objetivo de Iberdrola es asegurar la fidelidad del cliente, la confianza en la marca y seguir cultivando los lazos, y lo hacen

Revista de Comunicación de la SEECI. 2021, n 54, 119-143 
Moyaert, H.; Vangehuchten, L y Fernández-Vallejo, A.M. La estrategia de comunicación de RSC de Iberdrola en Facebook y Twitter: Un análisis lingüístico y de contenido basado en corpus

utilizando un lenguaje informal. Dado que el contenido de RSC de los informes a veces puede resultar difícil de leer o comprender por parte del público (Smeuninx, De Clerck \& Aerts, 2018), Iberdrola combina una comunicación seria de RSC con un lenguaje informal. Así podemos concluir que el lenguaje informal a la hora de comunicar sobre RSC sí fomenta la cercanía entre grupos de interés y marcas, precisamente porque la tasa de seguidores y engagement de Iberdrola es muy alta.

Los aspectos multimodales más destacados del corpus son los hashtags y las etiquetas (o "hipervínculos apelativos"), que también se codificaron al analizar la interactividad de las publicaciones de Iberdrola en las redes sociales. Según Catamutto \& Vela Delfa (2016), los hashtags son una especie de mecanismo que sirve para contrarrestar la tendencia efímera de los mensajes de las redes sociales. En otras palabras, ayudan a la recuperación ( $y$ a generar una cierta unidad textual) de publicaciones, que de otra manera son bastante temporales.

Por otro lado, los hipervínculos apelativos, o etiquetas, son un tipo especial de estrategia que consiste en hacer referencia al perfil de audiencia relevante para aumentar los cambios de un comentario. Vela Delfa (2017) afirma que este también es un elemento esencial en el discurso digital, porque esta hipertextualidad conduce a un discurso altamente coherente.

Si observamos la forma en que Iberdrola implementa estos elementos, podemos concluir que la firma utiliza ambas estrategias para mejorar el discurso digital y, por lo tanto, está utilizando estas características de las redes sociales para mejorar la búsqueda y el engagement (Cunha et al., En Zappavigna, 2015). El porcentaje de cobertura de los hashtags es alto, con un $37,56 \%$ en Twitter y un $81,01 \%$ en Facebook. Las etiquetas tienen un porcentaje de cobertura ligeramente menor, pero aún bastante alto, con un $13,01 \%$ en Twitter y un $16,22 \%$ en Facebook. Esto significa que Iberdrola considera ambos elementos esenciales.

\section{CONCLUSIÓN}

Como se expone en la sección 2, existe una discrepancia considerable entre la teoría de la RSC y la realidad, por lo cual las organizaciones no están seguras de la idoneidad de las redes sociales para la RSC, a menudo abogando por una combinación de medios (Postma, 2015). Además, existe confusión sobre el impacto de la comunicación de RSC en las redes sociales, porque algunos afirman que hay una tendencia a interactuar más con mensajes que no son de RSC que con mensajes de RSC, lo que podría deberse al hecho de que la mayoría de las publicaciones relacionadas con RSC utilizaron una estrategia informativa en lugar de interactiva, y las organizaciones no utilizan los beneficios que ofrecen las redes sociales (Cho, Furey \& Mohr, 2016). Otros afirman que la comunicación de RSC tiene un impacto más positivo que los productos/servicios, aunque se utiliza menos en las redes sociales (Etter, 2013). Además, las investigaciones afirman que los informes de RSC de las empresas europeas tienden a ser creíbles, pero mediocres, y que deben abordar diferentes temas para dirigirse a una audiencia de múltiples partes interesadas a fin de evitar el escepticismo y crear conciencia (Lock \& Seele, 2016).

Revista de Comunicación de la SEECI. 2021, nº 54, 119-143 
Moyaert, H.; Vangehuchten, L y Fernández-Vallejo, A.M. La estrategia de comunicación de RSC de Iberdrola en Facebook y Twitter: Un análisis lingüístico y de contenido basado en corpus

Aquí es donde surge la urgencia por el desarrollo de estrategias de comunicación de RSC para alentar al público a comprometerse con las corporaciones y varios aspectos de su RSC, al incluir a los públicos internos y externos involucrados en los programas (Cho et al., 2016). Por tanto, existe una gran necesidad de un marco de investigación que pueda proporcionar la base para una orientación práctica.

En este estudio contribuimos a este objetivo examinando la comunicación de RSC en redes sociales de Iberdrola, empresa española del IBEX 35 que puntúa bien tanto en información de RSC como en gestión de redes sociales, intentamos dar un claro ejemplo a seguir por otras empresas. Los resultados de PI1, "¿qué temas relacionados con la RSC se utilizan (con más frecuencia)?", sugieren que el enfoque de RSC de la estrategia de información de Iberdrola varía según el canal. Por un lado, Twitter se centra en el medio ambiente, la sostenibilidad, la inversión social, las partes interesadas y las artes. Por otro lado, la estrategia de información en Facebook está dirigida a los grupos de interés $y$, por lo tanto, a motivarlos en términos de involucrarse con información relacionada con la RSC. En ambos canales de redes sociales, los temas de RSC son ciertamente importantes, con el medio ambiente, la sostenibilidad y la inversión social predominantemente presentes, pero se informan de manera diferente. Por otro lado, los hipervínculos apelativos, o etiquetas, son un tipo especial de estrategia que consiste en hacer referencia al perfil de audiencia relevante para aumentar los cambios de un comentario. Vela Delfa (2017) afirma que este también es un elemento esencial en el discurso digital, porque esta hipertextualidad conduce a un discurso altamente coherente.

Los resultados de la PI2, "¿existe un posible patrón discursivo en la forma en que se formulan los mensajes?", sugieren que ciertamente existe una estrategia interactiva, con muchos elementos que caracterizan el discurso digital, y que ciertamente se aprovechan. Hay un uso claro de estructuras que provocan emociones (positivas), uso de lenguaje informal y elementos altamente discursivos, como hashtags y etiquetas. Las estructuras positivas, los emojis, el uso de $1^{\mathrm{a}}$ y $2^{\mathrm{a}}$ persona y los hashtags se encuentran entre los elementos más utilizados. Todos estos elementos son esfuerzos para aumentar la confianza, el engagement y la admiración de la marca.

Los resultados de la PI3, "¿se combinan ciertos temas con ciertos elementos textuales?", sugieren que existen algunos pequeños patrones subyacentes entre la estrategia de información y la estrategia de interacción, pero que la forma general en que Iberdrola quiere comunicar su información de RSC es a través de lenguaje emocional informal y (positivo). La única observación que se puede hacer es que el escaso uso de lenguaje emocional negativo siempre se combina con un tema ambiental, muy probablemente para evitar acusaciones de "greenwashing".

\section{RECOMENDACIONES}

Naturalmente, el estudio tiene sus limitaciones (por ejemplo, tamaño de la muestra, período de tiempo, etc.). Aunque se estudiaron las publicaciones de dos redes sociales y el tamaño de la muestra fue suficientemente representativo, solo se

Revista de Comunicación de la SEECI. 2021, nº 54, 119-143 
Moyaert, H.; Vangehuchten, L y Fernández-Vallejo, A.M. La estrategia de comunicación de RSC de Iberdrola en Facebook y Twitter: Un análisis lingüístico y de contenido basado en corpus

estudió en detalle una empresa energética del IBEX 35. Además, el período de tiempo de las publicaciones en las redes sociales fue durante un período de tres meses lleno de acontecimientos, con importantes feriados y conferencias sobre el clima. Un análisis longitudinal podría revelar si la comunicación cambia con el tiempo, lo que debería estar sujeto a más investigaciones. Un estudio que incluya retweets, favoritos y me gustas también podría proporcionar más información sobre el engagement con los diferentes tipos de contenido y elementos textuales, que ahora se basa simplemente en suposiciones. Todos estos elementos serían de gran relevancia en respuesta a la necesidad actual de un marco basado en la investigación sobre la comunicación de la RSC en las redes sociales.

\section{REFERENCIAS}

Beukeboom, C. J., Kerkhof, P., \& De Vries, M. (2015). Does a virtual like cause actual liking? How following a brand's Facebook updates enhances brand evaluations and purchase intention. Journal of Interactive Marketing, 32, 26-36. http://dx.doi.org/10.1016/j.intmar.2015.09.003 .

Brown, C., \& Nwagbara, U. (2018). Corporate Social Responsibility and Strategic Market Positioning for Organizational Success. https://books.google.be/books?id=d-NhDwAAQBAJ\&pg =PA80\&lpg=PA80\&dq

Bonsón, E. \& Ratkai, M. (2013). A set of metrics to assess stakeholder engagement and social legitimacy on a corporate Facebook page. Online Information Review, 37(5), 787-803.

Catamutto, L. y Vela Delfa, C. (2016). "El discurso digital como objeto de estudio: de la descripción de interfaces a la definición de propiedades". Aposta. Revista de Ciencias Sociales, 69, http://apostadigital.com/revistav3/hemeroteca/cvela2.pdf 296-323,

Cho, M., Furey, L. D., \& Mohr, T. (2016). Communicating Corporate Social Responsibility on Social Media. Business and Professional Communication Quarterly, 80(1), 52-69. https://doi.org/10.1177/2329490616663708

Coombs, W. T. \& Holladay, S. (2012). Privileging an Activist vs. A Corporate View of Public Relations History in the US. Public Relations Review, 38(3-, 347-353. https://doi.org/10.1016/j.pubrev.2011.11.010

De Choudhury, M., Sundaram, H., Lin, Y. R., John, A., \& Seligmann, D. D. (2009, June). Connecting content to community in social media via image content, user tags and user communication. In 2009 IEEE International Conference on Multimedia and Expo (pp. 1238-1241). IEEE. 
Moyaert, H.; Vangehuchten, L y Fernández-Vallejo, A.M. La estrategia de comunicación de RSC de Iberdrola en Facebook y Twitter: Un análisis lingüístico y de contenido basado en corpus

Du, S., Bhattacharya, C. B. \& Sen, S. (2010). Maximizing Business Returns to Corporate Social Responsibility (CSR): The Role of CSR Communication. Journal of Management Reviews, 12(1), 8-19.

Eberle, D., Berens, G., \& Li, T. (2013). The impact of Interactive Corporate Social Responsibility Communication on Corporate Reputation. Journal of Business Ethics, $118(4), 731-746$.

Epsilon. (2016, April 19). La presencia corporativa de las compañías del Ibex 35 en las redes sociales. Retrieved May 26, 2020. https://www.epsilontec.com/estudioibex-35-analitica-redes-sociales/

Etter, M. (2013). Reasons for low levels of interactivity (Non-) interactive CSR communication in twitter. Public Relations Review, 39, 606-608.

Fassin, Y., Van Rossem, A., \& Buelens, M. (2011). Small-business owner-managers' perceptions of business ethics and CSR-related concepts. Journal of Business Ethics, 98(3), 425-453.

Fieseler, C. \& Fleck, M. (2013). The pursuit of Empowerment through Social Media: Structural Social Capital Dynamics in CSR-Blogging. Journal of Business EthicS, $118(4), 759-775$.

Fournier, S., \& Avery, J. (2011). The uninvited brand. Business Horizons, 54, 193207. http://dx.doi.org/10.1016/j.bushor.2011.01.001.

Fernández-Vallejo, A. M. (2018). Metáforas y emociones en el twitter corporativo: una aproximación discursivo-lingüística a los microblogs de Acciona e Iberdrola. Círculo de Lingüística Aplicada a la Comunicación, 73(0). https://doi.org/10.5209/clac.59062

Fernández-Vallejo, A. M. (2020). Comunicar emociones en Facebook: Análisis discursivo en la página corporativo de la empresa Dia, Revista Española de Lingüistica Aplicada/Spanish Journal of Applied Linguistics, 33(1), 55-83. https://doi.org/10.1075/resla.17052.fer

Friedman, T. L. (2006). The world is flat: A brief history of the twenty-first century. New York: Farrar, Straus and Giroux.

Global Reporting. (n.d.). GRI Standards Download Homepage. Retrieved 30 March 2020. https://www.globalreporting.org/standards

Haigh, M. M., Brubaker, P., \& Whiteside, E. (2013). Facebook: examining the information presented and its impact on stakeholders. Corporate Communications: An International Journal, 18(1), 52-69. 
Moyaert, H.; Vangehuchten, L y Fernández-Vallejo, A.M. La estrategia de comunicación de RSC de Iberdrola en Facebook y Twitter: Un análisis lingüístico y de contenido basado en

Han, K. J. \& Chang-Hoan Cho. (2013). Twitter as a Tool for Corporate Communication: Exploring Interactive Communication Patterns between Fortune 500 Companies and Twitter Users. International Journal of Online Marketing (IJOM) 3(4), 68-83.

Iberdrola, named as one of the world's 100 most sustainable companies. (2019, January 22). Retrieved May 23, 2020. https://www.iberdrola.com/pressroom/news/detail/iberdrola-named-world-s-most-sustainable-companies

Iberdrola. (n.d.). Iberdrola, the Spanish company with the best corporate governance. Retrieved 12 February 2020, from https://www.iberdrola.com/pressroom/news/detail/iberdrola-spanish-company-with-best-corporate-governance

IBEX 35 Index. (n.d.). Retrieved May 23, 2020. https://capital.com/ibex-35- indexdefinition/

Keeling, K., Mcgoldrick, P., \& Beatty, S. (2010). Avatars as salespeople: Communication style, trust, and intentions. Journal of Business Research, 63, 793-800. http://dx.doi.org/10.1016/j.jbusres.2008.12.015 .

LibGuides: Statistical \& Qualitative Data Analysis Software: About NVivo. Retrieved May 26, 2020. https://libquides.library.kent.edu/statconsulting/NVivo/

Lock, I., \& Seele, P. (2016). The credibility of CSR (corporate social responsibility) reports in Europe. Evidence from a quantitative content analysis in 11 countries. Journal of Cleaner Production, 122, 186-200. https://doi.org/10.1016/j.jclepro.2016.02.060

McArthur, T. (1992). The Oxford companion to the English language. Oxford, UK: Oxford University Press.

Ministerio de Empleo y Seguridad Social. (2014). Spanish Corporate Social Responsibility Strategy. http://www.mitramiss.gob.es/ficheros/rse/documentos/eerse/EERSE-Inglesweb.pdf

Observatorio RSC. (2018). La responsabillidad social corporativa en las memorias anuales de las empresas del IBEX 35: análisis del Ejercicio 2018. https://observatoriorsc.org/descarga-gratis-el- informe/download-id/6753/

Postma, R. (2015). De Dichotomie van Sociale Media: Een Kloof tussen Theorie en Praktijk (Master's thesis). https://www.researchgate.net/profile/Rosa May Postma/publication/2759570 87 De Dichotomie van Sociale Media Een Kloof tussen Theorie en Praktij k/links/554b504c0cf21ed2135921bc.pdf 
Moyaert, H.; Vangehuchten, L y Fernández-Vallejo, A.M. La estrategia de comunicación de RSC de Iberdrola en Facebook y Twitter: Un análisis lingüístico y de contenido basado en corpus

Smeuninx, N., De Clerck, B., \& Aerts, W. (2018). Measuring the readability of sustainability reports: a corpus-based analysis through standard formulae and NLP. International Journal of Business Communication.

Van Noort, G., \& Willemsen, L. M. (2011). Online damage control: The effects of proactive versus reactive webcare interventions in consumer-generated and brand-generated platforms. Journal of Interactive Marketing, 26, 131-140. http://dx.doi.org/10.1016/j.intmar.2011.07.001 .

Van Zandvoort, E. (2016). Exercising power through CSR communication on Facebook: insights from the oil industry (Master's thesis). http://www.divaportal.org/smash/record.jsf?pid=diva2\%3A948818\&dswid=4355

Vela Delfa, C. (2017). Coherencia, cohesión y estructura de la interacción en el discurso digital: un análisis de los intercambios en la red social Facebook. In M. Giammatteo, A. Parini, \& P. Gubitosi (Eds.), El español en la red (pp. 255-278). Iberoamericana Vervuert.

Zappavigna, M. (2015). Searchable talk: the linguistic functions of hashtags. Social Semiotics, 25(3), 274-291. https://doi.org/10.1080/10350330.2014.996948

\section{AUTOR/ES:}

\section{Helena Moyaert}

Máster en Comunicación Profesional Multilingüe (junio 2020) y graduada en Lingüística Aplicada Español e Inglés (junio 2018) por la Universidad de Amberes, Bélgica. Interés especial en la comunicación empresarial digital de marketing. Sólidos conocimientos de administración y gestión de empresas y de lingüística aplicada. helena.moyaert@gmail.com

\section{Lieve Vangehuchten}

Lieve Vangehuchten es doctora en lenguas románicas por la Universidad Católica de Lovaina la nueva (Bélgica) y profesora titular de Español para Fines Académicos y Profesionales en la Facultad de Filosofía y Letras de la Universidad de Amberes (Bélgica). Es miembro fundador de CORESCO, un grupo de investigación internacional sobre la comunicación de la Responsabilidad Social Corporativa en el mundo hispánico: www.uantwerpen.be/coresco. Ha publicado varios artículos en este ámbito, así como en otras áreas de la lingüística aplicada al español. Su monografía El léxico del discurso económico empresarial: identificación, selección y enseñanza en español como lengua extranjera con fines espećficos (Vervuert/ Iberoamericana: Frankfurt/ Madrid, 2005, 776 p., serie Lingüística Iberoamericana, 26) fue muy bien recibida en los círculos académicos.

lieve.vangehuchten@uantwerpen.be

Orcid ID: https://orcid.org/0000-0002-6166-7701

Google Scholar: https://scholar.google.com/citations?user=hOdY4ZgAAAAJ\&hl=es

ResearchGate: https://www.researchgate.net/profile/Lieve Vangehuchten

Revista de Comunicación de la SEECI. 2021, nº 54, 119-143 
Academia.edu: https://antwerp.academia.edu/LieveVangehuchten

\section{Ana María Fernández Vallejo}

Ana M. Fernández-Vallejo, doctora, es miembro del Centro de Cultura y Sociedad (CCS) de la Universidad de Navarra (Pamplona, España) como colaboradora interna, y profesora asociada del Departamento de Comunicación de la Escuela de Asistentes de Dirección (Universidad de Navarra). Su investigación se centra en el estudio interdisciplinario del discurso académico y profesional. Sus intereses incluyen temas como la lingüística del discurso, la pragmática y la lingüística de corpus. En la actualidad, participa en proyectos relacionados con el lenguaje y la emoción en entornos profesionales y políticos a través de los medios digitales, y el discurso de RSC en los medios digitales. Es coorganizadora y miembro del comité científico de varias conferencias sobre comunicación empresarial y política. Además, es revisora frecuente de conferencias y revistas a doble ciego sobre idiomas con fines específicos.

amf@unav.es

Orcid ID: https://orcid.org/0000-0003-3877-651X

Google Scholar: https://scholar.google.es/citations?user=134Nuu4AAAAJ\&hl=es

ResearchGate: https://www.researchgate.net/profile/Ana Fernandez-Vallejo2

Academia.edu: https://navarra.academia.edu/anafernandez 